\title{
The Inclusion-Exclusion Principle On the Set of IF-sets
}

\author{
Jana Kelemenová \\ Faculty of Natural Sciences, Matej Bel University, Slovakia
}

\begin{abstract}
P. Grzegorzewski [3] has worked the probability version of the inclusion-exclusion principle and made a generalization for IF-events. He had applied two versions of the generalized formula, corresponding to different t-conorms and so defined the union of IF-events. This paper contains the generalization of the Grzegorzewski theorem. We prove it for mappings from the set of IF sets to the unit interval([2], [1]). Similar generalizations are presented in [4] and $[5]$.
\end{abstract}

Keywords: inclusion-exclusion principle, IF-sets, probability

\section{Introduction}

K. Atanassov introduced in [1] the notion of an IF - set as a mapping

$$
\begin{gathered}
A=\left(\mu_{A}, \nu_{A}\right) . \\
\mu_{A}, \nu_{A}: \Omega \rightarrow<0,1>
\end{gathered}
$$

are such that $\mu_{a}+\nu_{a} \leq 1, \mathcal{F}$ is the set of all IF -sets such that $A=\left(\mu_{A}, \nu_{A}\right)$. He considered also the following operations on $\mathcal{F}$ :

$$
\begin{aligned}
& A \cap B=\left(\mu_{A} \wedge \mu_{B}, \nu_{A} \vee \nu_{B}\right) \\
& =\left(\min \left(\mu_{A}, \mu_{B}\right), \max \left(\nu_{A}, \nu_{B}\right)\right) \\
& A \cup B=\left(\mu_{A} \vee \mu_{B}, \nu_{A} \wedge \nu_{B}\right) \\
& =\left(\max \left(\mu_{A}, \mu_{B}\right), \min \left(\nu_{A}, \nu_{B}\right)\right)
\end{aligned}
$$

for any $A=\left(\mu_{A}, \nu_{A}\right), B=\left(\mu_{B}, \nu_{B}\right) \in \mathcal{F}$.

P. Grzegorzewski in [3] considers a classical probability space $(\Omega, \mathcal{S}, \mathcal{P})$, when $\Omega$ is a non-empty set, $\mathcal{S}$ is a $\sigma$-algebra of subsets of $\Omega$, and $P: \mathcal{S} \rightarrow\langle 0,1\rangle$ is a probability measure, i.e. $\mathrm{P}$ is $\sigma$-additive and $\mathcal{P}(\Omega)=1$. He works with IF-events, that are such IF-sets $A=\left(\mu_{A}, \nu_{A}\right)$ that $\mu_{A}, \nu_{A}: \Omega \rightarrow\langle 0,1\rangle$ are $\mathcal{S}$ - measureable, i.e. $\mathcal{B} \subset R, \mathcal{B}$ is a Borel set $\Rightarrow \mu_{A}^{-1}(B) \in \mathcal{S}, \nu_{A}^{-1}(B) \in \mathcal{S}$.

P.Grzegorzewski considered the mapping $m$ : $\mathcal{F} \rightarrow\langle 0,1\rangle$, defined by the equality:

$$
\mathcal{P}(A)=\mathcal{P}\left(\mu_{A}, \nu_{A}\right)=\left(\int_{\Omega} \mu_{A} d P, 1-\int_{\Omega} \nu_{A} d P\right) .
$$

He extended the inclusion-exclusion principle for such mappings, i.e.

$$
\begin{aligned}
& \mathcal{P}\left(\bigcup_{i=1}^{n} A_{i}\right)=\sum_{k=1}^{n} \sum_{j_{1}, \ldots, j_{k}}(-1)^{k+1} m\left(A_{j_{1}} \cap \ldots \cap A_{j_{k}}\right), \\
& \text { e.g. } \\
& \qquad \begin{array}{r}
\mathcal{P}\left(A_{1} \cup A_{2}\right)=\mathcal{P}\left(A_{1}\right)+\mathcal{P}\left(A_{2}\right)-\mathcal{P}\left(A_{1} \cap A_{2}\right)(\mathbf{I}) \\
\text { or } \\
\qquad \mathcal{P}\left(A_{1} \cup A_{2} \cup A_{3}\right)=\mathcal{P}\left(A_{1}\right)+\mathcal{P}\left(A_{2}\right)+\mathcal{P}\left(A_{3}\right)- \\
\quad-\mathcal{P}\left(A_{1} \cap A_{2}\right)-\mathcal{P}\left(A_{1} \cap A_{3}\right)-\mathcal{P}\left(A_{2} \cap A_{3}\right)+ \\
\quad+\mathcal{P}\left(A_{1} \cap A_{2} \cap A_{3}\right)
\end{array}
\end{aligned}
$$

etc.

In the paper, we prove the inclusion-exclusion principle for any strongly additive mappings $m$ : $\mathcal{F} \rightarrow\langle 0,1\rangle$ i.e. mappings satisfying $(\mathbf{I})$. The result is a generalization of the result of [3]. E.g. the inex principle works for the mappings $m^{b}(A), m^{\sharp}(A)$ : $\mathcal{F} \rightarrow\langle 0,1\rangle$ defined by

$$
\begin{aligned}
& m^{b}(A)=\frac{1}{2} \int_{\Omega} \mu_{A} d P+\frac{1}{2} \int_{\Omega} \nu_{A} d P, \\
& m^{\sharp}(A)=\frac{3}{4} \int_{\Omega} \mu_{A} d P+\frac{1}{4} \int_{\Omega} \nu_{A} d P,
\end{aligned}
$$

hence also for $\mathcal{P}: \mathcal{F} \rightarrow\langle 0,1\rangle \times\langle 0,1\rangle$ defined by the equality

$$
\mathcal{P}(A)=\left(m^{b}(A), m^{\sharp}(A)\right) .
$$

Of course, the mapping cannot be covered by the Grzegorzewski result. Recall that another generalizations of [1] will be published in [4] and [5].

\section{Inclusion-exclusion principle for IF-sets}

Theorem 1 Let $\mathcal{F}$ be the set of pairs $A=\left(\mu_{A}, \nu_{A}\right)$;

$$
\begin{gathered}
A \leq B \\
\mu_{A} \leq \mu_{B}, \nu_{A} \geq \nu_{B} \\
0=(\boldsymbol{0}, \boldsymbol{1})
\end{gathered}
$$

$\mu_{A}, \nu_{A}: \Omega \rightarrow<0,1>, \mu_{A}+\nu_{A} \leq 1$. Let the mapping $m: \mathcal{F} \longrightarrow\langle 0,1\rangle$ be strongly additive, that is

$$
m(a \cup b)+m(a \cap b)=m(a)+m(b)
$$




$$
m(0)=0 \text {. }
$$

Then for $n$ even we have

$$
m\left(a_{1} \cup a_{2} \cup \ldots \cup a_{n}\right)+\sum_{k=1}^{n / 2} S_{2 k}^{(n)}=\sum_{k=1}^{n / 2} S_{2 k-1}^{(n)},
$$

where

$S_{k}^{(n)}=\sum_{1 \leq i_{1}<i_{2}<\ldots<i_{k} \leq n} m\left(a_{i_{1}} \cap a_{i_{2}} \cap \ldots \cap a_{i_{k}}\right)$.

and for $n$ odd we have

$$
\begin{gathered}
m\left(a_{1} \cup a_{2} \cup \ldots \cup a_{n}\right)+\sum_{k=1}^{(n+1) / 2-1} S_{2 k}^{(n)}= \\
=\sum_{k=1}^{(n+1) / 2} S_{2 k-1}^{(n)} .
\end{gathered}
$$

\section{Proof}

For the inclusion-exclusion principle on the IFsets the distributivity law holds:

$$
\begin{gathered}
(a \cap c) \cup(b \cap c)= \\
=\left(\left(\mu_{a} \wedge \mu_{c}\right) \vee\left(\mu_{b} \wedge \mu_{c}\right),\left(\nu_{a} \vee \nu_{c}\right) \wedge\left(\nu_{b} \vee \nu_{c}\right)\right)= \\
=\left(\mu_{a} \vee \mu_{b}\right) \wedge \mu_{c},\left(\nu_{a} \wedge \nu_{b}\right) \vee \nu_{c}=(a \cup b) \cap c .
\end{gathered}
$$

It holds also:

$$
c \cap c=\left(\mu_{c} \wedge \mu_{c}, \nu_{c} \vee \nu_{c}\right)=\left(\mu_{c}, \nu_{c}\right)=c .
$$

\subsection{If $n$ is even}

For $n$ even the induction assumption is

$$
\begin{array}{r}
m\left(\bigcup_{k=1}^{n} a_{k}\right)+ \\
+\sum_{k=1}^{n / 2} \sum_{1 \leq i_{1}<\ldots<i_{2 k} \leq n} m\left(a_{i_{1}} \cap \ldots \cap a_{i_{2 k}}\right)= \\
=\sum_{k=1}^{n / 2} \sum_{1 \leq i_{1}<\ldots<i_{2 k-1} \leq n} m\left(a_{i_{1}} \cap \ldots \cap a_{i_{2 k-1}}\right)
\end{array}
$$

From (1) we have

$$
\begin{aligned}
m\left(\left(\bigcup_{k=1}^{n} a_{k}\right) \cup a_{n+1}\right) & +m\left(\left(\bigcup_{k=1}^{n} a_{k}\right) \cap a_{n+1}\right)= \\
& =m\left(\bigcup_{k=1}^{n} a_{k}\right)+m\left(a_{n+1}\right)
\end{aligned}
$$

Moreover,

$$
m\left(\left(\bigcup_{k=1}^{n} a_{k}\right) \cap a_{n+1}\right)=m\left(\bigcup_{k=1}^{n}\left(a_{k} \cap a_{n+1}\right)\right)
$$

$$
\begin{array}{r}
m\left(\bigcup_{k=1}^{n}\left(a_{k} \cap a_{n+1}\right)\right)+ \\
+\sum_{k=1}^{n / 2} \sum_{1 \leq i_{1}<\ldots<i_{2 k} \leq n} m\left(\bigcap_{j=1}^{2 k} a_{i_{j}} \cap a_{n+1}\right)= \\
=\sum_{k=1}^{n / 2} \sum_{1 \leq i_{1}<\ldots<i_{2 k-1} \leq n} m\left(\bigcap_{j=1}^{2 k-1} a_{i_{j}} \cap a_{n+1}\right) .
\end{array}
$$

From (6) and by adding terms to both sides of equation we obtain :

$$
\begin{array}{r}
m\left(\bigcup_{k=1}^{n+1} a_{k}\right)+m\left(\bigcup_{k=1}^{n}\left(a_{k} \cap a_{n+1}\right)\right)+\sum_{k=1}^{n / 2} S_{2 k}^{(n)}+ \\
+\sum_{k=1}^{n / 2} \sum_{1 \leq i_{1}<\ldots<i_{2 k} \leq n} m\left(\bigcap_{j=1}^{2 k} a_{i_{j}} \cap a_{n+1}\right)= \\
=m\left(a_{n+1}\right)+m\left(\bigcup_{k=1}^{n} a_{k}\right)+\sum_{k=1}^{n / 2} S_{2 k}^{(n)}+ \\
+\sum_{k=1}^{n / 2} \sum_{1 \leq i_{1}<\ldots<i_{2 k} \leq n} m\left(\bigcap_{j=1}^{2 k} a_{i_{j}} \cap a_{n+1}\right)
\end{array}
$$

and so for the right side of equation by the induction assumption we have:

$$
\begin{array}{r}
m\left(a_{n+1}\right)+m\left(\bigcup_{k=1}^{n} a_{k}\right)+\sum_{k=1}^{n / 2} S_{2 k}^{(n)}+ \\
+\sum_{k=1}^{n / 2} \sum_{1 \leq i_{1}<\ldots<i_{2 k} \leq n} m\left(\bigcap_{j=1}^{2 k} a_{i_{j}} \cap a_{n+1}\right) \\
=m\left(a_{n+1}\right)+\sum_{k=1}^{n / 2} S_{2 k-1}^{(n)}+ \\
+\sum_{k=1}^{n / 2} \sum_{1 \leq i_{1}<\ldots<i_{2 k} \leq n}^{2 k} m\left(\bigcap_{j=1}^{2 k} a_{i_{j}} \cap a_{n+1}\right)
\end{array}
$$

By (8) and (9) we have

$$
\begin{array}{r}
m\left(\bigcup_{k=1}^{n+1} a_{k}\right)+\sum_{k=1}^{n / 2} S_{2 k}^{(n)}+ \\
+\sum_{k=1}^{n / 2} m\left(\bigcap_{1 \leq i_{1}<\ldots<i_{2 k-1} \leq n}^{2 k-1} a_{i_{j}} \cap a_{n+1}\right)= \\
=m\left(a_{n+1}\right)+\sum_{k=1}^{n / 2} S_{2 k-1}^{(n)}+ \\
+\sum_{k=1}^{n / 2} \sum_{1 \leq i_{1}<\ldots<i_{2 k} \leq n} m\left(\bigcap_{j=1}^{2 k} a_{i_{j}} \cap a_{n+1}\right)
\end{array}
$$


hence

$$
\begin{aligned}
& m\left(\bigcup_{k=1}^{n+1} a_{k}\right)+ \\
& +\sum_{k=1}^{(n+2) / 2-1} \sum_{1 \leq i_{1}<\ldots<i_{2 k} \leq n+1} m\left(\bigcap_{j=1}^{2 k} a_{i_{j}}\right)= \\
& =\sum_{k=1}^{(n+2) / 2} \sum_{1 \leq i_{1}<\ldots<i_{2 k-1} \leq n+1} m\left(\bigcap_{j=1}^{2 k-1} a_{i_{j}}\right) \text {. }
\end{aligned}
$$

So,

$m\left(\bigcup_{k=1}^{n+1} a_{k}\right)+\sum_{k=1}^{(n+2) / 2-1} S_{2 k}^{(n+1)}=\sum_{k=1}^{(n+2) / 2} S_{2 k-1}^{(n+1)}$.

\subsection{If $n$ is odd}

Let $\mathrm{n}$ be odd, hence the induction assumption gives

$m\left(\bigcup_{k=1}^{n} a_{k}\right)+\sum_{k=1}^{(n+1) / 2-1} S_{2 k}^{(n)}=\sum_{k=1}^{(n+1) / 2} S_{2 k-1}^{(n)}$.

Induction assumption implies

$$
\begin{array}{r}
m\left(\bigcup_{k=1}^{n}\left(a_{k} \cap a_{n+1}\right)\right)+ \\
+\sum_{k=1}^{(n+1) / 2-1} \sum_{1 \leq i_{1}<\ldots<i_{2 k} \leq n} m\left(\bigcap_{j=1}^{2 k} a_{i_{j}} \cap a_{n+1}\right)= \\
=\sum_{k=1}^{(n+1) / 2} \sum_{1 \leq i_{1}<\ldots<i_{2 k-1} \leq n} m\left(\bigcap_{j=1}^{2 k-1} a_{i_{j}} \cap a_{n+1}\right) .
\end{array}
$$

By the same proceeding as in (8), for $n$ odd we have:

$$
\begin{array}{r}
m\left(\bigcup_{k=1}^{(n+1)} a_{k}\right)+m\left(\bigcup_{k=1}^{n}\left(a_{k} \cap a_{n+1}\right)\right)+\sum_{k=1}^{n / 2} S_{2 k}^{(n)}+ \\
+\sum_{k=1}^{(n+1) / 2-1} \sum_{1 \leq i_{1}<\ldots<i_{2 k} \leq n} m\left(\bigcap_{j=1}^{2 k} a_{i_{j}} \cap a_{n+1}\right)= \\
=m\left(\bigcup_{k=1}^{n} a_{k}\right)+m\left(a_{n+1}\right)+\sum_{k=1}^{n / 2} S_{2 k}^{(n)}+ \\
+\sum_{k=1}^{(n+1) / 2-1} \sum_{1 \leq i_{1}<\ldots<i_{2 k} \leq n}^{2 k} m\left(\bigcap_{j=1} a_{i_{j}} \cap a_{n+1}\right) .
\end{array}
$$

By induction assumption and (11) and (12)

$$
\begin{aligned}
& m\left(\bigcup_{k=1}^{n+1} a_{k}\right)+\sum_{k=1}^{n / 2} S_{2 k}^{(n)}+ \\
& +\sum_{k=1}^{(n+1) / 2} \sum_{1 \leq i_{1}<\ldots<i_{2 k-1} \leq n} m\left(\bigcap_{j=1}^{2 k-1} a_{i_{j}} \cap a_{n+1}\right)+ \\
& =\sum_{k=1}^{n+1 / 2} S_{2 k-1}^{(n)}+m\left(a_{n+1}\right)+ \\
& \sum_{k=1}^{(n+1) / 2-1} \sum_{1 \leq i_{1}<\ldots<i_{2 k} \leq n} m\left(\bigcap_{j=1}^{2 k} a_{i_{j}} \cap a_{n+1}\right) .
\end{aligned}
$$

Hence,

$$
\begin{aligned}
& m\left(\bigcup_{k=1}^{n+1} a_{k}\right)+\sum_{k=1}^{(n+1) / 2} \sum_{1 \leq i_{1}<\ldots<i_{2 k} \leq n+1} m\left(\bigcap_{j=1}^{2 k} a_{i_{j}}\right)= \\
& =\sum_{k=1}^{(n+1) / 2} m\left(\bigcap_{j=1}^{2 k-1} a_{i_{j}} \cap a_{n+1}\right) . \\
& m\left(\bigcup_{k=1}^{(n+1)} a_{k}\right)+\sum_{k=1}^{(n+1) / 2} S_{2 k}^{(n)}=\sum_{k=1}^{(n+1) / 2} S_{2 k-1}^{(n)}
\end{aligned}
$$

\section{Examples}

Example 1 Let $a, b, c \in \mathcal{F}$. Then,

$$
m((a \cup b) \cap c)=m((a \cap c) \cup(b \cap c)),
$$

hence for $n=3$

$$
\begin{gathered}
m((a \cup b) \cup c)+m((a \cup b) \cap c)=m(a \cup b)+m(c) \\
m(a \cup b \cup c)+m(a \cap c \cup b \cap c)+m(a \cap b)= \\
=m(a \cup b)+m(c)+m(a \cap b)
\end{gathered}
$$

$m(a \cup b \cup c)+m(a \cap c \cup b \cap c)+m(a \cap b \cap c)+$

$+m(a \cap b)=m(a)+m(b)+m(c)+m(a \cap b \cap c)$

$$
\begin{array}{r}
m(a \cup b \cup c)+m(a \cap c)+m(b \cap c)+m(a \cap b)= \\
=m(a)+m(b)+m(c)+m(a \cap b \cap c)
\end{array}
$$

\section{Conclusions}

The classical inclusion- exclusion principle states that for any probability measure $\mathcal{P}:(\Omega, \mathcal{S}) \rightarrow\langle 0,1\rangle$ and any $A_{1}, \ldots, A_{n} \in \mathcal{S}$ holds

$$
\mathcal{P}\left(\bigcup_{i=1}^{n} A_{i}\right)=\sum_{i=1}^{n} P\left(A_{i}\right)-\sum_{i<j} P\left(A_{i} \cap A_{j}\right)+
$$


$+\sum_{i<j<k} \mathcal{P}\left(A_{i} \cap A_{j} \cap A_{k}\right)-\ldots+(-1)^{n+1} P\left(\bigcap_{i=1}^{n} A_{i}\right)$.

For strongly additive measures $m: \mathcal{S} \rightarrow<0,1>$ it holds:

$$
\begin{array}{r}
m\left(a_{1} \cup a_{2} \cup a_{3}\right)= \\
-m\left(a_{1}\right)+m\left(a_{2}\right)+m\left(a_{3}\right) \\
-m\left(a_{1} \cap a_{2}\right)-m\left(a_{1} \cap a_{3}\right)-m\left(a_{2} \cap a_{3}\right) \\
+m\left(a_{1} \cap a_{2} \cap a_{3}\right) . s e e[4]
\end{array}
$$

and similarly for any $m\left(a_{1} \cup a_{2} \cup \ldots \cup a_{n}\right)$. In this paper we generalize the principle for strongly additive states defined on the set of IF-sets.

\section{References}

[1] K. Atanassov, Intuitionistic Fuzzy Sets: Theory and Applications, Physica- Verlag, New York, 1999.

[2] B. Riečan, D. Mundici, Probability on MV algebras, Handbook of Measure Theory, Amsterdam, New York, pages 869 - 909, 2002.

[3] P. Grzegorzewski, The Inclusion-Exclusion Principle for IF - Events, Information Sciences, Volume 181, Issue 3, pages 536-546, 2011.

[4] M. Kuková, The Inclusion-Exclusion Principle for IF-events, to appear in Information Sciences, 2011.

[5] J. Kelemenová, The Inclusion-Exclusion Principle in semigroups. To appear in Developments in Fuzzy Sets, Intuitionistic Fuzzy Sets, Generalized Nets and Related Topics, proceedings of the $9^{\text {th }}$ international workshop on intuitionistic fuzzy sets and generalized nets (IWIFSGN 2010), IBS PAN - SRI PAS, Warsaw, 2011. 